\title{
Chiral symmetry restoration and the string picture of hadrons.
}

\author{
L. Ya. Glozman \\ Institute for Theoretical Physics, University of Graz, Universitätsplatz 5, A-8010 Graz, Austria*
}

\begin{abstract}
QCD string picture of highly excited hadrons very naturally explains parity doubling once the chiral symmetry is restored high in the spectrum. In particular, the spin-orbit and tensor interactions of quarks at the ends of the string, related to dynamics of the string, vanish. High in the spectrum there appears higher degree of degeneracy, namely parity doublets with different angular momentum cluster around energy of the string in the given quantum state.
\end{abstract}

It has recently been shown that the validity of the operator product expansion in QCD at large space-like momenta and the validity of the dispersion relation for the two-point correlator (i.e. validity of the Källen - Lehmann representation) implies a smooth chiral symmetry restoration in the high-energy part of hadron spectra composed of light quarks [1.2]. Indeed, at large space-like momenta $Q^{2}$ the difference between the two correlators $\Pi_{J_{1}}\left(Q^{2}\right)$ and $\Pi_{J_{2}}\left(Q^{2}\right)$ obtained with the local currents $J_{1}$ and $J_{2}$ which are connected by the chiral transformation $J_{1}=U J_{2} U^{\dagger}$, $U \in S U(2)_{L} \times S U(2)_{R}$ is due only to the small contributions of the chiral condensates that are suppressed by inverse powers of momenta,

$$
\Pi_{J_{1}}\left(Q^{2}\right)-\Pi_{J_{2}}\left(Q^{2}\right) \sim \frac{1}{Q^{n}}, n>0 .
$$

Since the large $Q^{2}$ correlator is completely dominated by the large $s$ spectral function, one expects that at large $s$ the theoretical spectral functions $\rho_{J_{1}}(s)$ and $\rho_{J_{2}}(s)$ must become essentially the same. This manifests a smooth chiral symmetry restoration from the low $s$ region, where both spectral functions are very different because of the chiral symmetry breaking in the vacuum, to the high $s$ region, where the chiral symmetry breaking in the vacuum becomes irrelevant.

Microscopically this is because the typical momenta of valence quarks should increase higher in the spectrum and once it is high enough the valence quarks decouple from the chiral condensates of the QCD vacuum and the dynamical (quasiparticle or constituent) mass of quarks drops off and the chiral symmetry gets restored [3]. This phenomenon does not mean that the spontaneous breaking of chiral symmetry in the QCD vacuum disappears, but rather that the chiral asymmetry of the vacuum becomes irrelevant sufficiently high in the spectrum. While the chiral symmetry breaking condensates in the vacuum are crucially important for the physics of the low-lying hadrons, the physics of the highly-excited states is such as if there were no chiral symmetry breaking in the vacuum.

This is in a very good analogy with the similar phenomenon in condensed matter physics. Namely, even if the metal is in the superconducting phase (i.e. there is a condensation of the Cooper pairs - which is analogous to the condensation of right-left quark pairs in the QCD vacuum - and the low-lying excitations of the system are the excitations of quasiparticles), the response of the superconductor to the high-energy (frequency) probe $\hbar \omega>>\Delta$ is the same as of the normal metal. This is because the phase coherent effects of the superconductor become irrelevant in this case and the external probe sees a bare particle rather than a quasiparticle. Similar, in the QCD case one has to probe the QCD vacuum by the high energy (frequency) external probe (current) in order to create a hadron of a large mass and hence the masses (physics) of the highly excited hadrons should be insensitive to the condensation of chiral pairs in the vacuum.

If high in the spectrum (i.e. where the chiral symmetry is approximately restored) the spectrum is still quasidiscrete, then the phenomenological manifestation of the chiral symmetry restoration would be that the highly excited hadrons should fall into the representations of the $S U(2)_{L} \times S U(2)_{R}$ group, which are compatible with the definite parity of the states - the parity-chiral multiplets [1,2]. In the case of baryons in the $N$ and $\Delta$ spectra these multiplets are either the parity doublets $\left((1 / 2,0) \oplus(0,1 / 2)\right.$ for $N^{*}$ and $(3 / 2,0) \oplus(0,3 / 2)$ for $\left.\Delta^{*}\right)$ that are not related to each other, or the multiplets $(1 / 2,1) \oplus(1,1 / 2)$ that combine one parity doublet in the nucleon spectrum with the parity doublet in the delta spectrum with the same spin. And indeed, the phenomenological highly-excited $N$ and $\Delta$ spectra

*e-mail: leonid.glozman@uni-graz.at 
show systematic parity doubling [1,2], though further experimental studies are required in order to make a definitive statement. It remains to be clarified which parity-chiral multiplets are observed - at the moment one cannot disentangle the different possibilities. In Fig. 1 we show the experimental $N$ spectrum [4]. Starting from $M \simeq 1.7 \mathrm{GeV}$ it is clear that the states systematically appear as approximate parity doublets, except for the two high-lying high-spin states, where the chiral partner have yet to be experimentally found. The onset of the approximate chiral symmetry restoration as low as in the region of $1.7 \mathrm{GeV}$ is very consistent with the recent finding of JLAB that the inclusive electroproduction of resonances in the mass region $3.1 \leq M^{2} \leq 3.9 \mathrm{GeV}^{2}$ is perfectly dual to deep inelastic data [0].

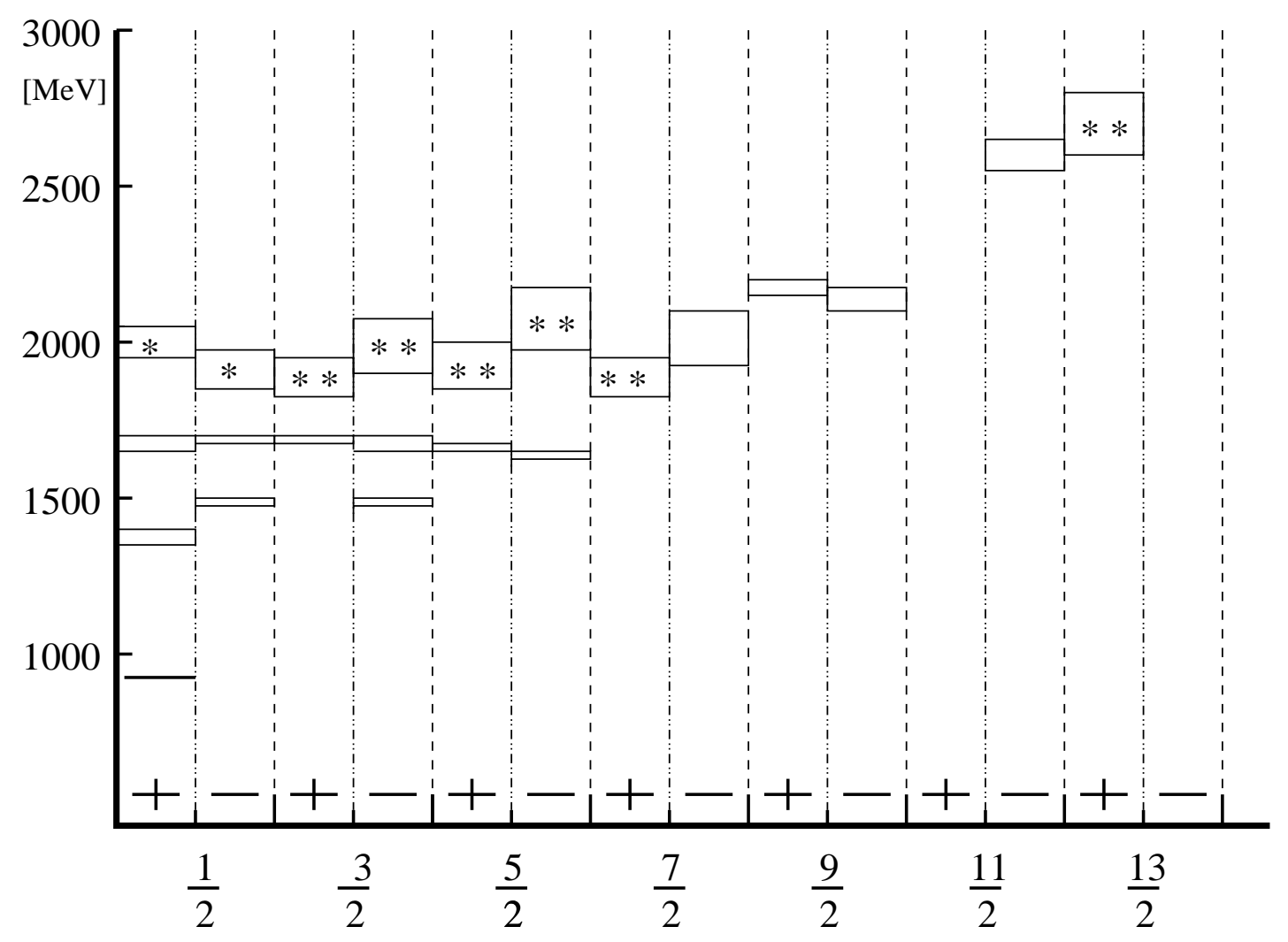

FIG. 1. Excitation spectrum of the nucleon. The real part of the pole position is shown. Boxes represent experimental uncertainties. Those resonances which are not yet reliably established are marked by two or one stars according to the PDG classification. The one-star resonances with $J=1 / 2$ around $2 \mathrm{GeV}$ are given according to the recent Bonn (SAPHIR) results $[8]$.

Systematic data on highly-excited mesons are still missing in the PDG tables. However, the data obtained from the ongoing partial wave analysis of mesonic resonances in the proton-antiproton annihilation at LEAR [6] show an almost systematic appearance of nearly perfect parity doublets in the meson spectrum in the mass region of $1.8 \mathrm{GeV}$ and above. A detailed analysis of the chiral multiplets with spin 0 is given in [8]. A similar situation is also observed for the highly excited mesons with higher spins. Yet the unique assignment of these higher spin states to the specific multiplets is difficult because sometimes the same state fits perfectly into different multiplets. For example, on the one hand the $\rho\left(1,1^{--}\right)$states at $1970 \pm 30 \mathrm{MeV}$ and at $2265 \pm 40 \mathrm{MeV}$ can be paired with the $a_{1}\left(1,1^{++}\right)$states at $1930_{-70}^{+30} \mathrm{MeV}$ and $2270_{-40}^{+55}$, respectively $((0,1) \oplus(1,0)$ representation). But one the other hand the same $\rho$-s can also be paired with $h_{1}\left(0,1^{+-}\right)$states at $1965 \pm 45$ and $2215 \pm 40$, respectively (i.e. the $(1 / 2,1 / 2)$ representation). Clearly a very systematic phase shift analysis should be done for both baryon and meson spectra and probably other properties such as decay modes should be invoked in order to unambiguously identify possible chiral partners.

Obviously, one also needs a physical picture for highly excited hadrons that is compatible with the chiral symmetry restoration and which would naturally explain the parity doubling. It is a purpose of this note to show that the QCD string picture of highly excited hadrons does this job. 
It is well known that the string picture very naturally incorporates the approximately linear behaviour of Regge trajectories for excited states. Low in the spectrum, where the spontaneous breaking of chiral symmetry in the vacuum is crucially important and which prevents the parity doubling, the linear behaviour of Regge trajectories is heavily broken [7]. In this part of the spectrum the physics which is associated with the quasiparticle picture (i.e. the constituent quark model) is more relevant and the effective interactions of quasiparticles can be approximated to some extent by potentials of different physical origin (e.g. by effective confining potential, flavor-spin potential which is associated with the Goldstone-boson-exchange between constituent quarks in the low-lying baryons, etc.).

Consider, as an example, the $\rho$ and $a_{1}$ mesons which become the chiral partners when the chiral symmetry is restored. The spectra of both types of mesons are extracted from the two-point functions

$$
\Pi_{\mu \nu}=\imath \int d^{4} x e^{\imath q x}\left\langle 0\left|T\left\{j_{\mu}(x) j_{\nu}^{\dagger}(0)\right\}\right| 0\right\rangle,
$$

where the currents (interpolating fields) for the isovector-vector and isovector-axialvector mesons are given as

$$
\begin{gathered}
j_{\mu}^{V}(x)=\bar{q}(x) \gamma_{\mu} \frac{\vec{\tau}}{2} q(x), \\
j_{\mu}^{A V}(x)=\bar{q}(x) \gamma_{\mu} \gamma_{5} \frac{\vec{\tau}}{2} q(x) .
\end{gathered}
$$

The chiral $S U(2)_{L} \times S U(2)_{R}$ transformations consist of the isospin as well as the axial transformations. The isospin transformations leave the currents above invariant while the axial transformations mix them

$$
j_{\mu}^{V}(x) \leftrightarrow j_{\mu}^{A V}(x) .
$$

Group theoretically both currents belong to the $(0,1) \oplus(1,0)$ representation of the parity-chiral group [2]. 1]

In the chiral limit both the vector and the axial-vector currents are conserved and the correlators for these currents can be written as

$$
\Pi_{\mu \nu}=\left(q_{\mu} q_{\nu}-q^{2} g_{\mu \nu}\right) \Pi\left(q^{2}\right) .
$$

The imaginary part of $\Pi\left(q^{2}\right)$ in the time-like domain $s=q^{2}>0$ is proportional to the spectral function $\rho(s)$. At large $s$ the spectral functions obtained with the currents (3) and (4) must coincide,

$$
\rho^{V}(s)=\rho^{A V}(s) .
$$

This manifests the chiral symmetry restoration. The theoretical spectral densities obtained in such a way can probably be compared with the experimental ones only upon some averaging [9]. For the present context the important thing is that if at large $s$ the spectrum is still quasidiscrete (i.e. it consists of separate resonances), then the equality of the vector and axial vector theoretical spectral densities is consistent with the approximate degeneracy of the highly excited vector and axial vector mesons. These mesons fill out in pairs the $(0,1) \oplus(1,0)$ representations of the parity-chiral group.

Microscopically this means the following. At large $s$ the valence quarks that are injected by the current into the vacuum do not become dressed by the quark condensates of the vacuum and remain left or right on the whole way of their propagation to the point where they are annihilated by the current. It is important to construct a model picture for the highly excited hadrons that is consistent with the given property of chiral symmetry restoration and that would explain chiral multiplets high in the hadron spectra.

\footnotetext{
${ }^{1}\left(I_{L}, I_{R}\right)$ denotes the irreducible representation of the chiral group with $I_{L}$ and $I_{R}$ being the isospins of the left and rigth quarks, respectively. Generally the irreducible representation of the chiral group is not invariant under parity, which transforms the left quarks into the right ones and vice versa. However, the direct sum of two irreducible representations $\left(I_{L}, I_{R}\right) \oplus\left(I_{R}, I_{L}\right)$ is invariant under parity and is an irreducible representation of the parity-chiral group.
} 
To carry out this task we will assume for the highly excited hadrons that the QCD string-like picture of confinement underlies the physics. We make the following simplifying assumptions: (i) the field in the string is of pure color-electric origin; (ii) the chiral symmetry is completely restored and hence the hadrons are rotating strings with massless quarks at the ends with definite chirality=helicity 1 and these valence quarks are combined into parity-chiral multiplets.

While for mesons the geometry of the string is rather obvious, it is not so for baryons. In the present context it is not so important whether the highly excited baryons are to be viewed as symmetric string configurations of the Mercedes-Benz type with the string junction in the center or are of a deformed type with the quark-diquark clustering. For us it is only important that the strings are pure electric and that the valence quarks at the ends have definite chirality=helicity and belong to one of the parity-chiral multiplets.

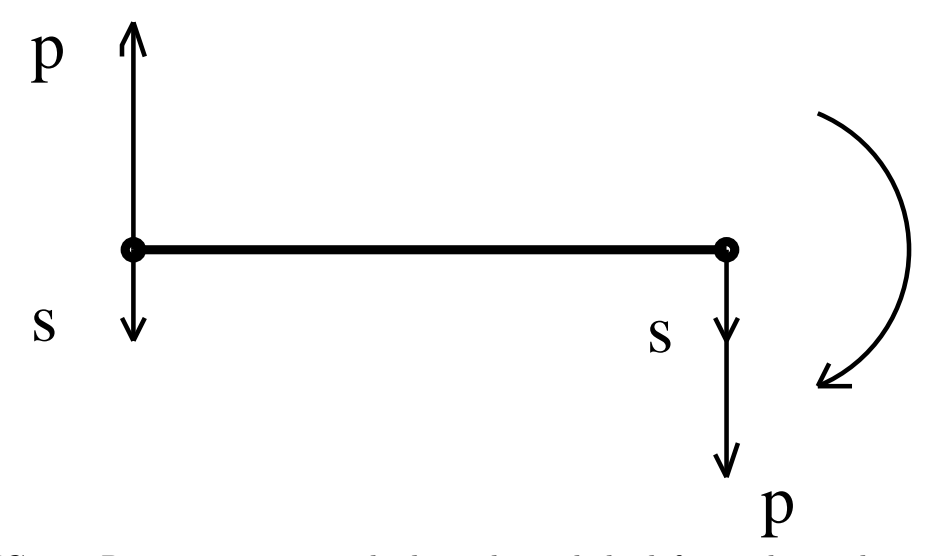

FIG. 2. Rotating string with the right and the left quarks at the ends.

The motion of the quarks at the ends of the string is constrained by the stationary motion of the string. This motion is a rotation. The key point is that massless $s=1 / 2$ quarks in the chirally restored regime possess a definite chirality=helicity (see Fig. 2). For the description of the rotation of massless quarks with definite chirality the angular momentum representation of the Weyl equations must be used, which for the stationary motion

$$
\Phi_{R, L}\left(j, j_{z}, t\right)=\phi_{R, L}\left(j, j_{z}\right) e^{-\imath E t}
$$

are

$$
\begin{gathered}
\vec{\sigma} \cdot \vec{\nabla} \phi_{R}\left(j, j_{z}\right)=\imath E_{R} \phi_{R}\left(j, j_{z}\right), \\
-\vec{\sigma} \cdot \vec{\nabla} \phi_{L}\left(j, j_{z}\right)=\imath E_{L} \phi_{L}\left(j, j_{z}\right) .
\end{gathered}
$$

Under parity transformation the Weyl equation for the right spinor is transformed into Weyl equation for the left spinor and vice versa. Since we require that the valence quarks belong to the same parity-chiral multiplet, then under parity operation the valence quarks must satisfy

$$
\phi_{L}\left(j, j_{z}\right) \leftrightarrow \phi_{R}\left(j, j_{z}\right) .
$$

One then obtains that the rotating string does not distinguish between the right and left valence quarks since they must have the same energy

$$
E_{L}=E_{R}
$$

Since the nonperturbative field in the string is pure electric, the magnetic interaction of quarks of the type $\vec{\sigma}(i) \cdot \vec{\sigma}(j)$ is absent. Though the string is pure electric, the quantum fluctuations of perturbative origin are possible once the

\footnotetext{
${ }^{2}$ This is a short notation for chirality $=+$ helicity for quarks and chirality $=-$ helicity for antiquarks.
} 
quarks are close to each other ${ }^{3}$ However, for massless quarks with definite chirality=helicity the interaction induced by perturbative forces, does not distinguish between the members of the parity-chiral multiplet.

The electric field in the string is "flavor-blind" and hence does not distinguish between the light quarks of different flavor once the chiral limit is taken.

Since chiral symmetry is assumed to be restored, the valence quarks are decoupled from the Goldstone bosons and hence the Goldstone boson mediated forces (e.g. the flavor-spin forces, that are crucially important in the low-lying baryons [11]) between quarks are also absent.

One arrives at the following situation: (i) the hadrons with the different chiral configurations of the quarks at the ends of the string which belong to the same parity-chiral multiplet and that belong to the same intrinsic quantum state of the string must be degenerate; (ii) the total parity of the hadron is determined by the product of parity of the string in the given quantum state and the parity of the specific parity-chiral configuration of the quarks at the ends of the string. There is no analogy to this situation in nonrelativistic physics where parity is only determined by the orbital motion of particles. Thus one sees that for every intrinsic quantum state of the string there necessarily appears parity doubling of the states with the same total angular momentum.

The spin-orbit operator $\vec{\sigma} \cdot \vec{L}$ does not commute with the helicity operator $\vec{\sigma} \cdot \vec{\nabla}$. Hence the spin-orbit interaction of quarks with the fixed chirality=helicity is absent. In particular, this is also true for the spin-orbit force due to the Thomas precession

$$
U_{T}=-\vec{\sigma} \cdot \vec{\omega}_{T} \sim \vec{\sigma} \cdot[\vec{v}, \vec{a}] \sim \vec{v} \cdot[\vec{v}, \vec{a}]=0,
$$

where $U_{T}$ is the energy of the interaction and $\vec{\omega}_{T}, \vec{v}$ and $\vec{a}$ are the angular frequency of Thomas precession, velocity of the quark and its acceleration, respectively.

In addition, for the rotating string

$$
\begin{aligned}
& \vec{\sigma}(i) \cdot \vec{R}(i)=0, \\
& \vec{\sigma}(i) \cdot \vec{R}(j)=0,
\end{aligned}
$$

where the indices $i, j$ label different quarks and $\vec{R}$ is the radius-vector of the given quark in the center-of-mass frame. The relations above immediately imply that the possible tensor interactions of quarks related to the string dynamics should be absent, once the chiral symmetry is restored.

As an example, consider a relativistic potential description of $\rho$ and $a_{1}$ mesons within the constituent quark model [12]. With this description the parity of the state is unambiguously prescribed by the relative orbital angular momentum of the quark and antiquark and the $\rho$ and $a_{1}$ mesons are the ${ }^{3} S_{1}$ and ${ }^{3} P_{1}$ states, respectively. Clearly, such a picture cannot explain the systematical degeneracy of $\rho$ and $a_{1}$ mesons high in the spectrum because stronger centrifugial repulsion and weaker spin-spin force in $a_{1}$ mesons systematically shifts them with respect to $\rho$ mesons. As a result, while the fitting of the parameters of the model provides success in the description of the low-lying $\rho$ mesons, the model completely fails in the description of $a_{1}$ mesons. Up to $2.4 \mathrm{GeV}$ it predicts only two $a_{1}$ states (at $1.24 \mathrm{GeV}$ and $1.82 \mathrm{GeV}$ ) while the experimental data indicates the states at $1.26 \mathrm{GeV}, 1.64 \mathrm{GeV}, 1.93 \mathrm{GeV}$ and 2.27

\footnotetext{
${ }^{3}$ Depending on whether the nonperturbative resummation of gluonic exchanges or the instanton-induced interactions provide the chiral symmetry breaking in QCD, the corresponding forces automatically split the low-lying mesons where chiral symmetry breaking effects are central to the physics.

${ }^{4}$ The coupling of Goldstone bosons to quasiparticles (constituent quarks) [10] is regulated by the Goldberger-Treiman relation taken at the quark level, $g=\frac{M g_{A}}{f_{\pi}}$, where $M$ is the quasiparticle (dynamical) mass of quarks induced by the coupling of quarks to the quark condensates. Chiral symmetry restoration high in the spectrum means (as it was explained above) that the valence quarks decouple from the quark condensates which in turn implies that their quasiparticle mass $M$ approaches to the current mass value (which is zero in the chiral limit). Hence the Goldstone bosons decouple from such valence quarks. This is in contrast to the low-lying hadrons where the chiral symmetry breaking effects are very important and where the coupling of valence quarks to the chiral condensates is crucial for physics.
} 


\section{$\mathrm{GeV}[6]$.}

The failure of the potential description is inherently related to its inability to incorporate chiral symmetry restoration. In contrast, in the string picture once the chiral symmetry is restored both $\rho$ and $a_{1}$ mesons belong in pairs to the same quantum state of the string with the same angular momentum of the string. The opposite parity of these states is provided by the different right-left configurations of valence quarks. While the vector mesons contain the following valence quark configuration

$$
\frac{1}{\sqrt{2}}\left(\bar{R} \frac{\vec{\tau}}{2} \gamma^{\mu} R+\bar{L} \frac{\vec{\tau}}{2} \gamma^{\mu} L\right)
$$

the axial vector mesons are constructed as

$$
\frac{1}{\sqrt{2}}\left(\bar{R} \frac{\vec{\tau}}{2} \gamma^{\mu} R-\bar{L} \frac{\vec{\tau}}{2} \gamma^{\mu} L\right) .
$$

Both hadrons actually are the different parity states of the same basic particle. This is like the $\rho^{+}, \rho^{0}, \rho^{-}$which represent different charge (isospin) states of the same particle. While isospin symmetry (which is not broken in the vacuum) is a good symmetry both for low-lying and high-lying hadrons, chiral symmetry (which contains isospin as a subgroup) is a good symmetry only high in the spectrum, in fact. So high in the spectrum it would even be proper to use the same "name" for chiral partners.

As was shown above, in the string picture once the chiral symmetry is restored the spin-orbit force is absent. Indeed, the phenomenological meson and baryon spectra in the $u, d$ sector do not show large spin-orbit forces. In contrast, in the potential picture the states are affected by the large spin-orbit force which comes both from the one gluon exchange interaction and the Thomas precession of quarks in the scalar confining potential. While for some specific family of mesons the fine tuning of parameters can provide an approximate cancellation of these strong spin-orbit interactions, this is not the case for the other family of mesons. For example, though the ${ }^{3} S_{1}$ states in $\rho$-s are not affected by the spin-orbit force, the spin-orbit force is strong in $a_{1}$ mesons within this description.

Once the quantum fluctuations of perturbative origin are neglected, the energy of the hadron is determined exclusively by the energy of the string; the mutial orientation of the spins of the quarks become irrelevant. In this case there appears an even higher degree of degeneracy than the simple parity doubling. Namely those hadrons with the same intrinsic energy and angular momentum of the string but with different total spin of all quarks (that are allowed by Pauli principle) must be degenerate. The higher we are in the spectrum - the smaller is the amplitude for the quarks to be close to each other and hence the perturbative contributions become suppressed. In other words, one should expect an appearance of sets of degenerate parity-doublets with different total angular momentum (i.e. clusters of parity-doublets) in hadron spectra. For example, if the intrinsic angular momentum of the string in an excited nucleon is $\mathrm{L}=1$, then the cluster of parity doublets should consist of three doublets with total angular momenta $J=1 / 2,3 / 2,5 / 2$ (because the total spin of three quarks can range from $1 / 2$ to $3 / 2$ ). The first cluster of parity doublets in the nucleon spectrum at $M \simeq 1.7 \mathrm{GeV}$ neatly fits this picture. If one assumes that the next excitation of the string is associated with $L=2$, then there should be cluster of parity doublets with $J=1 / 2,3 / 2,5 / 2,7 / 2$. And indeed the band of baryons in the $N$ and $\Delta$ spectra in the mass region $M \simeq 2 \mathrm{GeV}$ does contain such parity doublets. The empirical parity doublets in meson spectrum with different total angular momentum also perfectly group into two clusters around the masses of $\simeq 2 \mathrm{GeV}$ and of $\simeq 2.2-2.3 \mathrm{GeV}$.

Clearly, in reality the chiral symmetry restoration is only approximate and also the masses of light quarks are not zero, so the helicity is not equal to chirality and the quark spin can flip with some small amplitude during the rotation of the string. It can also be not parallel to the quark momentum. Consequently there must appear weak spin-spin, spin-orbit and tensor interactions of different origins between the valence quarks. These weak interactions should give rise to the small splittings in parity doublets as well as among different doublets within the cluster. It is a challenging task to evaluate such corrections.

I am grateful to D.V. Bugg for providing me with data [6] on highly excited meson spectra. This work was supported by the FWF Project P14806-TPH. 
[1] T. D. Cohen and L. Ya. Glozman, Phys. Rev. D65 (2002) 016006

[2] T. D. Cohen and L. Ya. Glozman, Int. J. Mod. Phys. A17 (2002) 1327.

[3] L. Ya. Glozman, Phys. Lett. B475 (2000) 329.

[4] Particle Data Group, D. Groom et al, Eur. Phys. J. C15 (2000) 1.

[5] I. Niculescu et al, Phys. Rev. Lett. 85 (2000) 1186.

[6] A. V. Anisovich et al, Phys. Lett. B491 (2000) 47; B517 (2001) 261; preprint "Combined analysis of meson channels with $\mathrm{I}=1, \mathrm{C}=-1$ from 1940 to $2410 \mathrm{MeV}$ "; preprint " $\mathrm{I}=0, \mathrm{C}=-1$ mesons from 1940 to $2410 \mathrm{MeV}$.

[7] L. Ya. Glozman, hep-ph/0105225.

[8] L. Ya. Glozman, Phys. Lett. B539 (2002) 257.

[9] E. C. Poggio, H. R. Quinn, and S. Weinberg, Phys. Rev. D13 (1976) 1958.

[10] A. Manohar and H. Georgi, Nucl. Phys. B234 (1984) 189.

[11] L. Ya. Glozman and D. O. Riska, Phys. Rep. 268 (1996) 263.

[12] S. Godfrey and N. Isgur, Phys. Rev. D32 (1985) 189.

[13] R. Plötzke et al, Phys. Lett. B444 (1998) 555. 